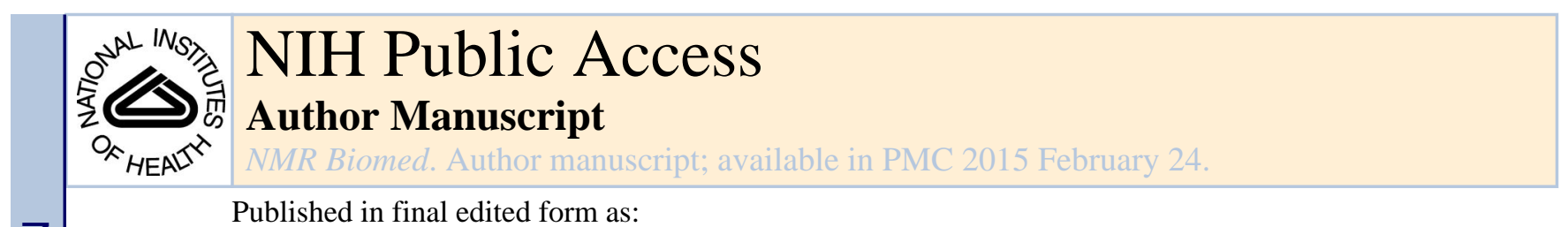

Published in final edited form as:

NMR Biomed. 2011 July ; 24(6): 559-560. doi:10.1002/nbm.1750.

\title{
Tumor microenvironment in cancer treatment and metastasis
}

\author{
N. R. Jagannathan and \\ Department of NMR and MRI Facility, All India Institute of Medical Sciences, New Delhi 110029, \\ India
}

Zaver M. Bhujwalla

Department of Radiology, The Johns Hopkins University School of Medicine, Baltimore, MD 21205, USA

N. R. Jagannathan: jagan1954@hotmail.com; Zaver M. Bhujwalla: zaver@mri.jhu.edu

The tumor microenvironment (TME), which consists of the vasculature, stromal cells, immune cells and the extracellular matrix, together with abnormal physiological environments, such as hypoxia and acidic extracellular $\mathrm{pH}\left(\mathrm{pH}_{\mathrm{e}}\right)$, influences a range of phenotypic traits of cancer, including invasion, metastasis and response to therapy.

The TME is increasingly occupying 'center stage' in providing new insights in cancer progression and treatment (Fig. 1). Stromal and immune elements also represent attractive therapeutic targets. An understanding of the complex relationships between cancer cells, stromal cells, immune cells and the extracellular matrix is limited. Furthermore, as interactions between cancer cells and the TME change continually with growth or therapy, the ability to noninvasively image the TME is critically important. Noninvasive imaging provides both the spatial and temporal resolution to understand and evaluate the continually changing dynamics of the TME with tumor growth and following treatment.

The TME provides a rich area to mine for the development of imaging biomarkers to evaluate response to treatment. A better understanding of the TME may lead to the development of better diagnostic tests and improved therapeutic strategies. The assessment of the tumor response to various treatments is an important aspect of patient treatment planning and management. As a result of the toxicity of chemotherapeutic agents, an early prediction of the therapeutic response is essential to avoid unnecessary toxicity in nonresponders.

With a redundancy of pathways and compensatory mechanisms, which include multiple interactions with the TME and stromal and immune cells, the cancer cell continues to evade and survive treatment strategies that incorporate advances of the 21st century. A single perturbation within a cancer cell or its microenvironment can create a cascade of changes in multiple pathways and networks. Importantly, in this era of targeted molecular medicine and 'omics', noninvasive molecular and functional imaging can be used to evaluate the response to personalized molecular-targeted treatments.

Copyright (C) 2011 John Wiley \& Sons, Ltd. 
This special issue of NMR in Biomedicine presents a collection of 14 primarily review articles from leading groups in the field that cover bench-to-bedside applications of imaging to: (i) understand the microenvironment of tumors and its influence on invasion, metastasis and therapeutic response; (ii) guide therapeutic targeting of the TME; and (iii) detect response to treatment. Although most of the review articles focus on MRI and MRS, some also include multimodality imaging approaches. This collection of review articles also provides molecular and functional imaging insights into invasion and metastasis.

The first six review articles are more discovery oriented and start with a focus on the use of imaging to investigate the TME and progress to the exploitation of the TME for the development of image-guided treatment strategies. In the first article, McCann et al. provide a comprehensive review of molecular imaging of tumor invasiveness and metastases, within the context of the advantages and disadvantages of MR-based methods. Next, Reut $e t$ al. detail the role of hypoxic stress in cancer, and review currently available techniques to image hypoxia. The abnormal physiological environment of tumors is further reviewed by Hashim et al. in an article that describes the role of the acidic $\mathrm{pH}_{\mathrm{e}}$ of tumors in the promotion of metastasis. Also reviewed in this article are current MR methods for the measurement of tumor $\mathrm{pH}$. The role of the TME on MR-visible lipids is reviewed by Delikatny et al. This article illustrates the impact of hypoxia, acidic $\mathrm{pH}_{\mathrm{e}}$ and inflammatory cells on the formation of mobile lipids. The use of mobile lipids as biomarkers to assess response to treatment is also discussed by the authors. The next two reviews discuss the TME and therapy. Bell et al. highlight the use of MR applications to investigate the TME, with a focus on emerging clinically translatable methods. Stasinopoulos et al. outline the characteristics of the TME, describe multimodal theranostic agents currently available to target the TME, and discuss the opportunities provided by the TME for the design of novel theranostic agents for cancer therapy.

These articles are followed by two review articles that focus on the use of MRS and MRSI in the characterization of tumor metabolism, the targeting of tumor metabolism and the use of metabolism to evaluate response to treatment. Podo et al. focus their review on cancer cells, detailing the aberrant choline metabolism that exists in cancer cells and reviewing the effect of tumor microenvironmental factors, such as hypoxia and acidic $\mathrm{pH}$, as well as molecular targeting, on choline and glucose metabolism. The use of MRS/I in understanding choline and glucose metabolism in tumors in vivo and in intact tissue using high-resolution magic angle spinning is reviewed by Glunde $e t$ al. Both of these reviews summarize the molecular underpinnings of the aberrant choline and glucose metabolism observed in cancers, their use in designing novel treatment strategies in cancer, and the development of biomarkers of cancer treatment using MR-detectable metabolites in choline and glucose metabolism.

The last six articles focus on human cancers and cover prostate, breast, liver, brain and musculoskeletal soft tissue tumors. Kayvan et al. present data that relate choline phospholipid metabolite concentrations to Gleason score, cellular proliferation and surgical stage in malignant prostatectomy specimens. Next, the use of ${ }^{1} \mathrm{H}$ MRS to detect the response to treatment in breast cancer patients, and some of the challenges associated with these applications, are reviewed by Sharma et al. McLaughlin and Hylton describe applications of 
dynamic contrast-enhanced MRI, diffusion-weighted MRI and ${ }^{1} \mathrm{H}$ MRS as early treatment predictors for response to neoadjuvant therapy in breast cancer and in staging residual disease. The applications of MRI and MRS in evaluating hepatic tumor response are described by Lee et al. This is followed by a review of the applications of MRI and MRS in brain tumor diagnosis, treatment planning and assessment of response to therapy by Nelson. Finally, Wang et al. review the applications of MRI and MRS in the diagnostic and therapeutic challenges presented by soft tissue sarcomas.

These articles highlight the existing and potential wealth of information that can be obtained on the TME and on the interactions between stromal, immune and cancer cells with imaging. They also illustrate the complexity, diversity and fluidity of the TME. Challenges for the future are to recognize the heterogeneity and complexity of the TME and to apply systems biology approaches to understand and exploit the TME for the development of effective treatments for cancer. MRI and MRS/I have tremendous strengths in functional imaging, but the lack of sensitivity of detection imposes some barriers for applications that require high sensitivity. A multimodality molecular functional imaging approach will provide new insights into the interactions between the cancer cell and its microenvironment.

Finally, we are grateful to the authors who readily agreed to contribute to this special issue, and commend them for providing an overview of current applications and future developments. Thanks are also due to various reviewers for sparing their valuable time in reviewing the articles. Special thanks are extended to Chris Boesch and John Griffiths for readily agreeing to publish this special issue. The valuable time and constant help provided by Chris Boesch are gratefully acknowledged. 


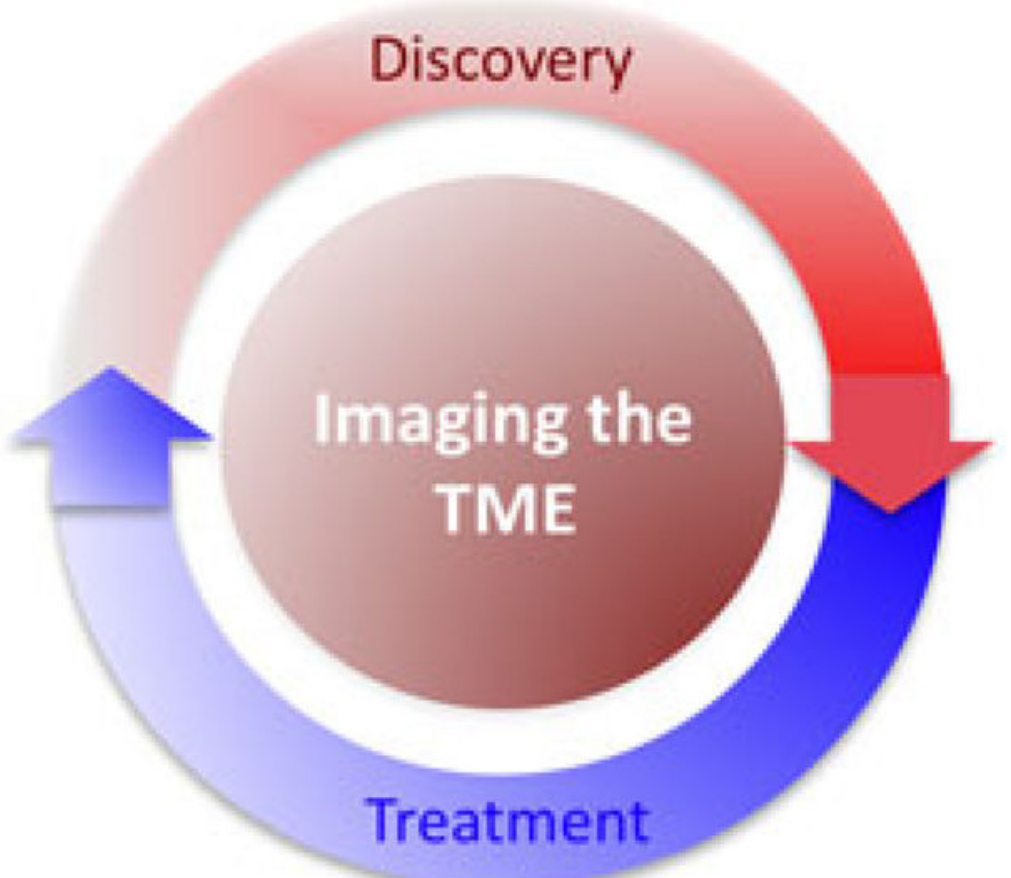

Figure 1.

Imaging the tumor microenvironment (TME) drives discovery and treatment in cancer. 